\title{
BECKWITH WAIDEMANN SYNDROME
}

\author{
By Seni Jeremiah (MD4 2005/2006)
}

\begin{abstract}
A five days old $3.3 \mathrm{~kg}$ infant, preterm male was admitted at the neonatal ward MNH five hours after birth from a peripheral hospital for further management of congenital hernia and general care. Examinations revealed macroglosia, earlobe crease, large exomphalous, small penis, hypospadius and cryptochidism. Investigations portrayed neonatal hypoglycaemia and distended bowel by $x$-ray. The ultimate diagnosis reached was Beckwith Waidemann Syndrome. The management for such a condition is mainly supportive to maintain euglycaemia, surgical intervention when indicated followed by monitoring the possibility of embryonal neoplasm later by ultrasonography surveillance.
\end{abstract}

\section{INTRODUCTION}

Congenital anomalies are structural defects present at birth. They may be inherited or sporadic, isolated or multiple, apparent or hidden, gross or microscopic. They cause nearly half of all deaths in term newborns. A major anomaly is apparent at birth in 3\% to $4 \%$ of newborns while up to $7.5 \%$ of children manifest a congenital defect by the age of 5 years ${ }^{1}$.

In 1964, Hans-Rudolf Wiedemann reported a familial form of omphalocele with macroglossia in Germany. In 1969, J. Bruce Beckwith of Loma Linda University, Calif, described a similar series of patients. Originally, Professor Wiedemann coined the term EMG syndrome to describe the combination of congenital exomphalos, macroglossia, and gigantism. Over time, this constellation was renamed BeckwithWiedemann syndrome ${ }^{2}$.

Beckwith Waidemann syndrome is a congenital anomaly which in most cases appears to be sporadic $(85 \%)$, though familial inheritance (autosomal dominance) has been reported. The aetiology of BWS is generally accepted as genetic ${ }^{3}$.The genetic defect has been shown to be as a result of chromosomal mutation (abnormal expression of gene) at $11 \mathrm{p} 15$, a region which also encodes the insulin like growth factor (IGF-2) and also very close to the Wilms tumour gene located at $11 \mathrm{p} 13$. There is paternal disomy for the segment containing IGF-2. Duplication of the above region and genetic imprint from defective or absent copy of the maternally derived gene are involved in variable features and patterns of transmission ${ }^{4}$.

The incidence has been reported to vary from 1 in 13,700 to 1 in 15,000 with no sex predisposition. Perinatal BWS is associated with high incidence of prematurity (up to $50 \%$ in some studies). The true incidence may go unreported because less severely affected cases are not reported or are misdiagnosed merely as abdominal wall defects or as infants who are large for their gestational age . $^{5}$.

There is also limited information on BWS at Muhimbili National Hospital (MNH) and Tanzania at large. As far as publications are concerned, throughout my clerkship in a neonatal ward for about three weeks I have seen four newborns with the syndrome, including the one I'm reporting.

\section{CASE}

A five days $3.3 \mathrm{~kg}$ male Muslim preterm (36/40 of gestation age) infant of M.O.H was admitted at the MNH neonatal ward from a peripheral hospital for further management of congenital umbilical hernia and general care five (5) hours after birth by hospital SVD on $21 / 1 / 2006$ at $5.00 \mathrm{pm}$ from a 35 years old mother Para $2+0$ (living issues 2). During delivery the mother sustained a minor tear which was sutured. The Apgar score at 5 and 10 minutes after birth was $6 / 10$ and $8 / 10$ respectively.

The mother experienced abdominal pain throughout the course of pregnancy at an approximate interval of $2-3$ weeks, the other findings in the course of pregnancy, labour and delivery were uneventful. The baby was breastfed (BF) after 3hours then referred to $\mathrm{MNH}$ where it was not $\mathrm{BF}$ for the next three days and was on I/V drips and oxygen. Cord had not healed (was still wet) and was cleaned with normal saline and dressed with paraffin gauze on alternative days. 
The baby's immunization and developmental milestones were uneventful.

The index was the second born to the mother and first to the father, who were not married but cohabiting. At delivery the mother was 35 years old, a primary school graduate and a hairdresser. The father was 45 years old, also a primary school graduate and a driver. The $1^{\text {st }}$ born was 18 years old female, had completed standard VII and was doing well. The mother was divorced by the firstborn's father in 1990. No congenital/hereditary diseases were reported from both paternal and maternal sides.

On examination, the baby was alert, floppy with a very weak cry, macrostomia and macroglossia. His weight was $3.3 \mathrm{~kg}$, length $48 \mathrm{~cm}$, OFC $35 \mathrm{~cm}$ and MUAC $8 \mathrm{~cm}$. All these were normal anthropometric measurements. He had a slightly distended abdomen with visible veins, big exomphalos approximately $8 * 10 \mathrm{~cm}$ with a very thin covering and visible viscera under the thin sac which was protruding as the baby cries. Tympanic percussion note and bowel sound were normal, he had a small penis ( $1 \mathrm{~cm}$ long), hypospadias and cryptorchidism with patent anus. There were no signs of organomegaly.

The baby was conscious, could suck and his tongue protruded centrally, other findings were non contributory.

Investigations requested were RBG which showed hypoglycaemia $(2.2 \mathrm{mmol} / \mathrm{l})$, Abdominal X-ray which showed distended bowel and abdominal ultrasonography which showed no enlargement of liver, kidney and spleen. Chromosome studying (Karyotyping) was also supposed to be undertaken but unfortunately was not a procedure done at the hospital.

On the day of admission the baby being preterm was given I/V 10\% dextrose $200 \mathrm{mls}$ in 24 hourly, vitamin $\mathrm{K}$ injection 0.5 $\mathrm{mg}$ stat, I/V ampiclox $165 \mathrm{mg} 12$ hourly for $10 / 7$ and I/V gentamycin $16.5 \mathrm{mg} 12$ hourly for $7 / 7$. The umbilicus was cleaned with normal saline and dressed with paraffin gauze on alternative days. EBM $10 \mathrm{mls} 3$ hourly was administered then increased on successive days. The baby was kept warm, vital signs were monitored along with nursing care and a consultation note was written to the paediatric surgeon.
After about two weeks of admission the baby was doing well, In the ward, blood glucose level were stabilized, the wound was clean with granulation tissue(sign of healing) and its size decreased (3*5). The paediatric surgeon recommendation revealed no indication for surgical repair of the defective abdominal wall. Generally the condition was stable.

\section{DISCUSSION}

BWS is a congenital anomaly in which the chromosome $11 \mathrm{p} 15$ is mutated. This is usually sporadic ( $85 \%)$, a state that is also true to this patient as no familial inheritance was noted (reported). The clinical findings vary widely and tend to become less obvious with age and because of this the syndrome may be overlooked in adults.

This is a disorder of growth characterized by macrosomia, macroglossia, visceromegaly, omphalocele, neonatal hypoglycaemia and increased susceptibility to embryonal tumours such as; Wilms tumour, hepatoblastoma, neuroblastoma and rhabdomyosarcoma ${ }^{5}$. Other features include hypospadias and cryptorchidism. Typical facial features include earlobe crease, prominent occiput, and nevus flammeus marked on the forehead, eyelid and neck, which is also called "angle's kiss". The minor features include microcephaly, congenital cleft palate, polycythemia and hyperinsulinaemia. Of these features, the baby I'm reporting presented with macroglossia, exomphalos, hypospadias, cryptorchidism, ear lobe crease and had neonatal hypoglycaemia.

Hypoglycaemia associated with hyperinsulinaemia is seen in approximate $50 \%$ of patients with BWS $^{5}$. Untreated neonatal hypoglycaemia is an important complication and may result in further cerebral dysfunction, such as seizures, mild to moderate mental retardation, and/or neonatal death in more severe cases. Macroglossia can cause variable complications ranging from feeding difficulties to airway obstruction and death. Neonatal mortality rate is approximately $21 \%{ }^{7}$. This child had also developed neonatal hypoglycaemia $(2.2 \mathrm{mmol} / \mathrm{l})$ which was fortunately stabilized by I/V $10 \%$ dextrose. Though the tongue was big the child could suck normally.

Wilms tumour of the kidney occurs in 7 out of 100 of those with hemi-hypertrophy and 
1 in 100 in those without. Other literature have shown that the risk of developing Wilms tumour regardless of hemihypertrophy state is around 7- $10 \%$, thus abdominal US and monitoring alpha fetoprotein every 3months until 5 years and then annually up to 10 years will help to unravel the possible abdominal tumour ${ }^{6}$, to this patient, if present will be revealed later as the child grows.

Perinatal BWS is associated with a relatively high incidence of prematurity $(50 \%$ of infants in some studies), ranging from 30 to 38 weeks. This was also true to the baby being reported as he was premature (36/40 of the gestation age). Polyhydramnios which occurs in $50 \%$ of the pregnancies may reflect impaired swallowing due to macroglossia or excess urine production from hypertrophied kidneys. The large placenta, large foetal size, and the polyhydramnios may result into early delivery due to lack of space in the uterus. This prematurity contributes to infant mortality that may be as high as $21 \%$. Causes of death include congestive heart failure, sleep apnoea (macroglossia), and infantile RDS ${ }^{5}$. These were not found in the patient.

The management of BWS is mainly supportive, though in some cases surgical intervention may be required to repair the defective abdominal wall, macroglossia and later for embryonal tumours. The goal of therapy is maintenance of plasma glucose above $60 \mathrm{mg} / \mathrm{dL}$ at all times by frequent feeding or diazoxide.

Prognosis in BWS depends primarily on the status of the airway and on aggressive prevention of hypoglycaemia, later on prognosis will depend on the embryonal neoplasm. The prognosis of this child was good as there was no further rebound hypoglycaemia and the healing response of the wound was good. There was still a concern on the possibility of developing neoplasms particularly Wilms tumour, thus a need of abdominal US for monitoring.
The parents were counselled on the genetical nature of the condition, maintenance of euglycaemia and the possibility of some embryonal neoplasms which though are not the rule of thumb.

I present this child because though BWS occur rarely; its management requires strict care as apart from the surgical intervention to correct the defective abdominal wall, most children die of neonatal hypoglycaemia and sepsis, conditions which can be intervened. The correction of the exomphalos, follow up in monitoring the alpha fetoprotein, surveillance ultrasonography and surgical management of the embryonal neoplasms pose a great challenge to the health professionals especially in the resource constrained setting like ours.

\section{REFERENCES}

1. Congenital anomalies. The Merck Manual of Diagnosis and Therapy. Chapter 261. http//www. merck.com.

2. G:\eMedicine - Beckwith-Wiedemann Syndrome Article by Robert J Ferry, Jr, MD.htm. Last Updated: January 13, 2005

3. Myrna R. Nieves, MD FAAP. G: \Wiedemann Beckwith Syndrome.htm. www.ibisbirthdefects.org

4. Behrman, Kliegman, Jenson. Nelson Textbook of Paediatric $17^{\text {th }}$ ed .Thomson Press (India) Ltd 2004.pg 512, 616, 1712, 1861.

5. Rosanna Weksberg, PhD, MD, et al. (March 3, 2000)). G:(Wiedemann Beckwith Syndrome.htm. www.ibis-birthdefects.org

6. William Sinclair. Hand book of Genetic and Congenital Syndromes. Oxford University Press 2005.

7. Sandra R. Silva, MD, et al. G: \Wiedemann Beckwith Syndrome.htm. Www.ibisbirthdefects.org 